\title{
Complete genome sequence of Stackebrandtia nassauensis type strain (LLR-40K-21 $1^{\mathrm{T}}$ )
}

\author{
Chris Munk ${ }^{1}$, Alla Lapidus ${ }^{2}$, Alex Copeland ${ }^{2}$, Marlen Jando ${ }^{3}$, Shanmugam Mayilraj ${ }^{3,4}$, Tijana \\ Glavina Del Rio' ${ }^{2}$, Matt Nolan' ${ }^{2}$, Feng Chen ${ }^{2}$, Susan Lucas ${ }^{2}$, Hope Tice ${ }^{2}$, Jan-Fang Cheng ${ }^{2}$, Cliff \\ Han $^{2,1}$, John C. Detter ${ }^{2,1}$, David Bruce ${ }^{2,1}$, Lynne Goodwin ${ }^{2,1}$, Patrick Chain ${ }^{2,1}$, Sam Pitluck ${ }^{2}$, \\ Markus Göker ${ }^{3}$, Galina Ovchinikova ${ }^{2}$, Amrita Pati ${ }^{2}$, Natalia Ivanova ${ }^{2}$, Konstantinos Mavro- \\ matis $^{2}$, Amy Chen ${ }^{5}$, Krishna Palaniappan ${ }^{5}$, Miriam Land ${ }^{2,6}$, Loren Hauser ${ }^{2,6}$, Yun-Juan \\ Chang $^{2,6}$, Cynthia D. Jeffries ${ }^{2,6}$, Jim Bristow ${ }^{2}$, Jonathan A. Eisen ${ }^{1,7}$, Victor Markowitz ${ }^{5}$, Philip \\ Hugenholtz $^{2}$, Nikos C. Kyrpides ${ }^{2}$, and Hans-Peter Klenk ${ }^{3 *}$ \\ ${ }^{1}$ Los Alamos National Laboratory, Bioscience Division, Los Alamos, New Mexico, USA \\ ${ }^{2}$ DOE Joint Genome Institute, Walnut Creek, California, USA \\ ${ }^{3}$ DSMZ - German Collection of Microorganisms and Cell Cultures GmbH, Braunschweig, \\ Germany \\ ${ }^{4}$ Microbial Type Culture Collection, Institute of Microbial Technology, Chandigarh, India \\ ${ }^{5}$ Biological Data Management and Technology Center, Lawrence Berkeley National Labora- \\ tory, Berkeley, California, USA \\ ${ }^{6}$ Oak Ridge National Laboratory, Oak Ridge, Tennessee, USA \\ ${ }^{7}$ University of California Davis Genome Center, Davis, California, USA
}

*Corresponding author: Hans-Peter Klenk

Keywords: aerobic, Gram-positive, non-acid-fast, mycelium producing, 2-hydroxy fatty acidscontaining, Glycomycetaceae

Stackebrandtia nassauensis Labeda and Kroppenstedt (2005) is the type species of the genus Stackebrandtia, and a member of the actinobacterial family Glycomycetaceae. Stackebrandtia currently contains two species, which are differentiated from Glycomyces spp. by cellular fatty acid and menaquinone composition. Strain LLR-40K- $21^{\top}$ is Gram-positive, aerobic, and nonmotile, with a branched substrate mycelium and on some media an aerial mycelium. The strain was originally isolated from a soil sample collected from a road side in Nassau, Bahamas. Here we describe the features of this organism, together with the complete genome sequence and annotation. This is the first complete genome sequence of the actinobacterial suborder Glycomycineae. The 6,841,557 bp long single replicon genome with its 6487 protein-coding and 53 RNA genes is part of the Genomic Encyclopedia of Bacteria and Archaea project.

\section{Introduction}

Strain LLR-40K-21 ${ }^{\mathrm{T}}$ (=DSM $44728=$ NRRL B$16338=$ JCM 14905) is the type strain of Stackebrandtia nassauensis, which is the type species of the genus Stackebrandtia [1]. S. nassauensis was originally isolated by M. P. Lechevalier and subsequently described by Labeda and Kroppenstedt [1] during the course of a $16 \mathrm{~S}$ rRNA survey of putative Glycomyces strains. The genus was named after Erko Stackebrandt, a German microbiologist of note, who has contributed significantly to the molecular systematics the Actinobacteria. At present the genus Stackebrandtia contains only one addi- tional species: S. albiflava, isolated from a soil sample collected from a tropical rainforest in China [2] .Here we present a summary classification and a set of features for $S$. nassauensis strain LLR$40 \mathrm{~K}-21^{\mathrm{T}}$ together with the description of the complete genomic sequencing and annotation.

\section{Classification and features}

A search of GenBank revealed no 16S rRNA reference sequences that were closely related to $S$. nassauensis. With 95\% sequence similarity, the type 
strain S. albiflava, YIM 45751 [2], is the only cultivated strain with a sequence similarity above $91 \%$, whereas a 16S rRNA gene sequence derived from a sample isolated from pig slurry (pig saw dust spent bedding in France, M982657, Snell-Castro et al., unpublished), represents the only related phylotype (with the same degree of sequence similarity as YIM 45751). Curiously, the type strains of the neighboring genus Glycomyces [3] were not within the 250 top hits in BLAST searches, with the $16 \mathrm{~S}$ rRNA of type species G. harbinensis [3] sharing only $89 \%$ sequence similarity. Screening of envi- ronmental genomic samples and surveys reported at the NCBI BLAST server also showed no closely related phylotypes (with 93\% sequence identity at the maximum), indicating a rather limited environmental occurrence of the species $S$. nassauensis (as of July 2009).

Figure 1 shows the phylogenetic neighborhood of $S$. nassauensis in a $16 \mathrm{~S}$ rRNA based tree. The two $16 \mathrm{~S}$ rRNA gene sequences in the genome of strain LLR$40 \mathrm{~K}-21^{\mathrm{T}}$ are identical and do not differ from the previously published $16 \mathrm{~S}$ rRNA sequence generated from NRRL B-16338 (AY650268).

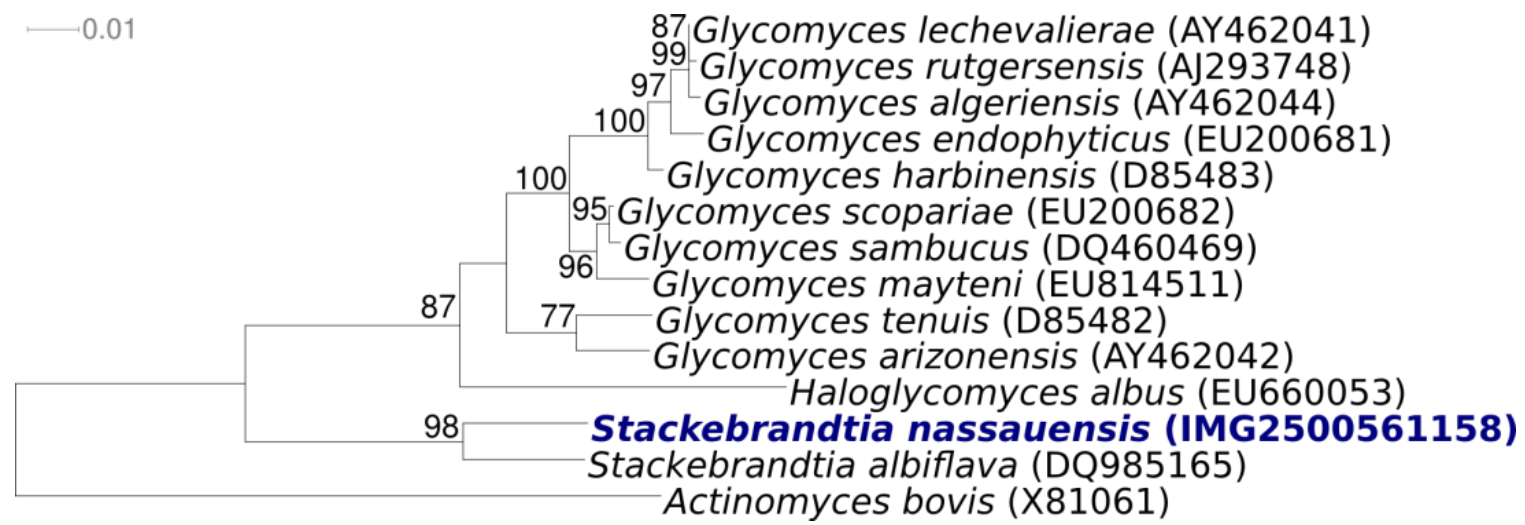

Figure 1. Phylogenetic tree of $S$. nassauensis strain LLR-40K- $21^{\top}$ and all type strains of the family Glycomycetaceae, inferred from 1,390 aligned characters [4] of the 16S rRNA sequence under the maximum likelihood criterion [5,6]. The tree was rooted with Actinomyces bovis, the type strain of the order Actinomycetales. The branches are scaled in terms of the expected number of substitutions per site. Numbers above branches are support values from 1,000 bootstrap replicates if larger than $60 \%$. Lineages with type strain genome sequencing projects registered in GOLD [7] are shown in blue, published genomes in bold.

S. nassauensis strain LLR-40K-21 $1^{\mathrm{T}}$ cells are nonmotile and filamentous, producing a with pale yellow to pale tan substrate mycelium on solid media [1] (Table 1 and Figure 2). Aerial mycelia are produced on some media and are white to yellowish-white in color [1]. Both aerial and substrate mycelia are approximately $0.5 \mu \mathrm{m}$ in diameter [1]. Fragmentation of aerial or substrate mycelia into chlamydospores or zoospores has not been observed [1]. Cells stain Gram-positive, grow aerobically, and are non acid-fast [1]. Growth occurs at the temperature range of $15-37^{\circ} \mathrm{C}$ and in the presence of $4-9 \% \mathrm{NaCl}$. S. nassauensis LLR-40K$21^{\mathrm{T}}$ is positive for hydrolysis or degradation of allantoin, casein, esculin, gelatin, hypoxanthine, starch and tyrosine but negative for adenine and xanthine [1]. The strain is capable of producing phosphatase and reducing nitrates; assimilation of acetate and malate is possible but not of benzoate, citrate, lactate, mucate, oxalate, propionate, succinate and tartarate [1]. Acid is produced aerobically from arabinose, cellobiose, dextrin, fructose, galactose, glucose, glycerol, lactose, maltose, mannose, melibiose, methyl $\alpha$-D-glucoside, raffinose, rhamnose, salicin, sorbitol, sucrose, trehalose and xylose; but not from adonitol, dulcitol, erythritol, inositol, mannitol, melezitose or methyl- $\beta$ xyloside [1]. 


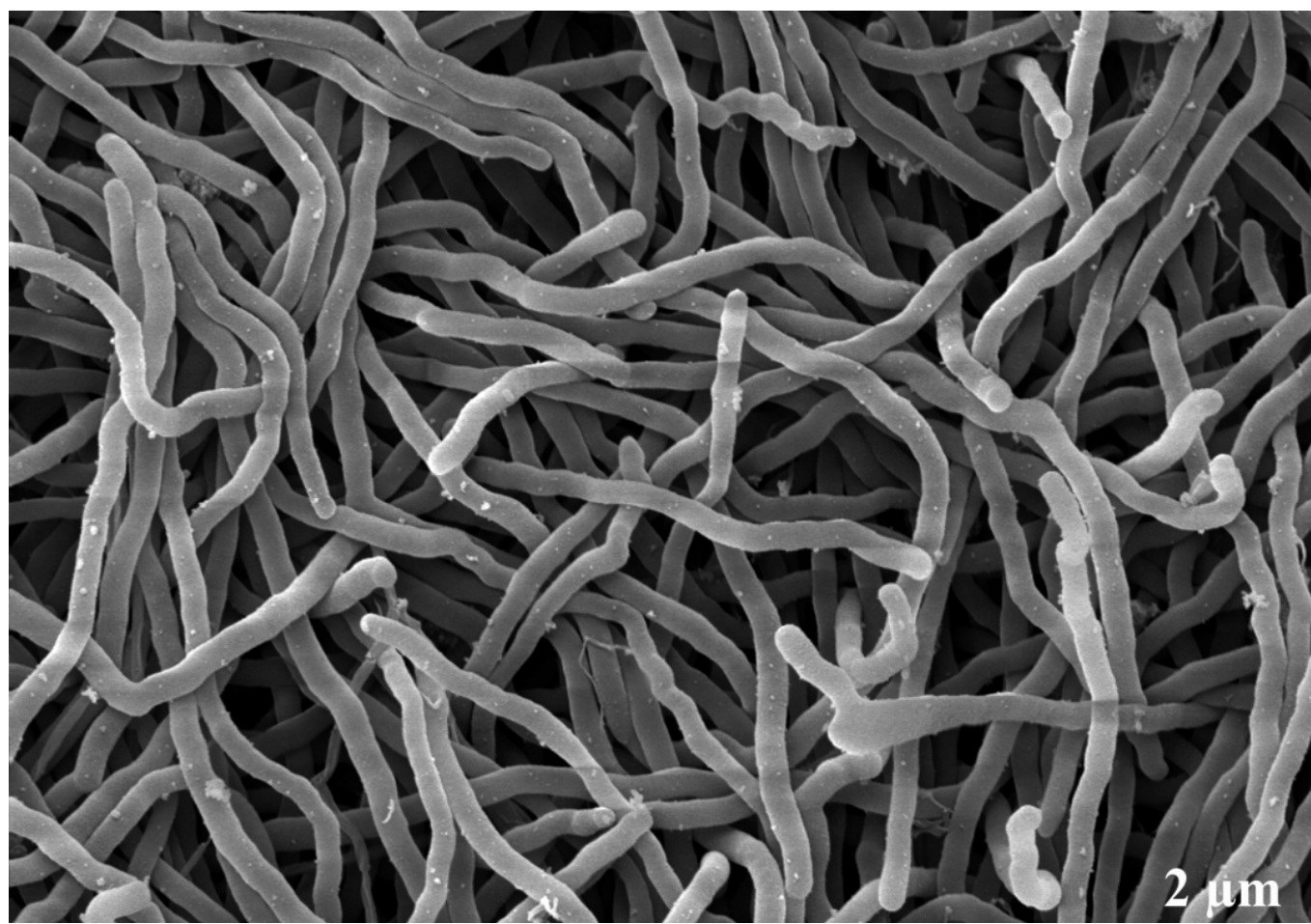

Figure 2. Scanning electron micrograph of $S$. nassauensis strain LLR-40K- $21^{\top}$ (Manfred Rohde, Helmholtz Centre for Infection Research, Braunschweig)

\section{Chemotaxonomy}

The murein of $S$. nassauensis strain LLR-40K-21 contains meso-diaminopimelic acid as the diamino acid and N-glycolylmuramic acid as is found in members of the genus Glycomyces. Ribose is the major cell wall sugar. Mannose has also been reported [1,2]. Reports about the presence of inositol, arabinose, xylose and glucose differ [1,2]. Galactose, which has been identified in all Glycomyces strains, has not been found in Stackebrandtia [1]. The fatty acid pattern of LLR-40K- $21^{\mathrm{T}}$ is dominated by saturated branched chain acids, anteiso-(ai-) $\mathrm{C}_{17: 0}$ (26.8\%), ai- $\mathrm{C}_{15: 0}$ (2.8\%), and iso-(i-) $\mathrm{C}_{15: 0}$ (8.7\%), i- $\mathrm{C}_{16: 1}(2.1 \%), \mathrm{i}-\mathrm{C}_{16: 0}(8.7 \%), \mathrm{i}-\mathrm{C}_{17: 0}(9.0 \%)$. Unsaturated straight chain acids play only a limited role: $\mathrm{C}_{17: 1 \text { cis9 }}(1.8 \%)$, and $\mathrm{C}_{16: 1 \text { cis9 }}(3.1 \%)$. $\mathrm{A}$ significant amount of ai- $\mathrm{C}_{17: 0} 2-\mathrm{OH}(14.5 \%)$ and moderate amounts of hydroxylated fatty acids were also detected. Moderate amounts of saturated components including 10-methyl-branched heptadecanoic acid $\mathrm{C}_{16: 0} 10$ methyl (9.0\%) and 10methyl- $\mathrm{C}_{17: 0}(1.4 \%)$ were also detected. The occurrence of 10-methyl branched heptadecanoic acid and i-branched 1-OH fatty acids is differential for S.nassauensis from members of the genus Glycomyces which lack these acids. Polar lipids identified are phosphatidylglycerol, diphosphatidylglycerol, like in members of the genus Glycomyces, and two additional yet unknown phospholipids are present. Phosphatidylinisitolmanosides (PIM) and phosphatidylglycerol (PI), which are present in the members of the genus Glycomyces, are absent; however, PI is present in S. albiflava [2]. Phosphatidylethanolamine (PE) and phosphatidylmethyl-ethanolamine (PME) were initially reported as absent in strain LLR-40K-21 ${ }^{\mathrm{T}}$ [1], but were later observed by Wang et al. [2]. The predominant menaquinones are MK-10 $\left(\mathrm{H}_{4}\right), \mathrm{MK}-10$ $\left(\mathrm{H}_{6}\right), \mathrm{MK}-11\left(\mathrm{H}_{4}\right)$ and MK-11 $\left(\mathrm{H}_{6}\right)$, different from the patterns observed from the members of the genus Glycomyces which contain menaquinones with 9-12 isoprene units with various degrees of hydrogenation [1]. Mycolic acids are absent [1].

\section{Genome sequencing and annotation}

\section{Genome project history}

This organism was selected for sequencing on the basis of its phylogenetic position, and is part of the Genomic Encyclopedia of Bacteria and Archaea project. The genome project is deposited in the Genomes OnLine Database [7] and the complete genome sequence in GenBank. Sequencing, finishing and annotation were performed by the DOE Joint Genome Institute (JGI). A summary of the project information is shown in Table 2. 
Table 1. Classification and general features of $S$. nassauensis strain LLR-40K- $21^{\top}$ according to the MIGS recommendations [8]

\begin{tabular}{|c|c|c|c|}
\hline MIGS ID & Property & Term & Evidence code \\
\hline & \multirow{8}{*}{ Current classification } & Domain Bacteria & TAS [9] \\
\hline & & Phylum Actinobacteria & TAS [10] \\
\hline & & Class Actinobacteria & TAS [11] \\
\hline & & Order Actinomycetales & TAS [11] \\
\hline & & Suborder Glycomycineae & TAS [11] \\
\hline & & Family Glycomycetaceae & TAS $[1,11,12$ \\
\hline & & Genus Stackebrandtia & TAS [1] \\
\hline & & $\begin{array}{l}\text { Species Stackebrandtia nassauensis } \\
\text { Type strain LLR-40K- } 21\end{array}$ & TAS [1] \\
\hline & Gram stain & positive & TAS [1] \\
\hline & Cell shape & hyphae, aerial and substrate mycelium & TAS [1] \\
\hline & Motility & non-motile & TAS [1] \\
\hline & Sporulation & non-sporulating & TAS [1] \\
\hline & Temperature range & mesophilic & TAS [1] \\
\hline & Optimum temperature & $15-37^{\circ} \mathrm{C}$ & TAS [1] \\
\hline & Salinity & $4-9 \mathrm{~g} \mathrm{NaCl} / \mathrm{l}$ & TAS [1] \\
\hline \multirow[t]{3}{*}{ MIGS-22 } & Oxygen requirement & aerobic & TAS [1] \\
\hline & Carbon source & glucose, maltose, mannose, cellobiose & TAS [1] \\
\hline & Energy source & starch & TAS [1] \\
\hline MIGS-6 & Habitat & soil & TAS [1] \\
\hline MIGS-15 & Biotic relationship & free-living & NAS \\
\hline \multirow[t]{3}{*}{ MIGS-14 } & Pathogenicity & none & NAS \\
\hline & Biosafety level & 1 & TAS [13] \\
\hline & Isolation & road side soil & TAS [1] \\
\hline MIGS-4 & Geographic location & Nassau, Bahamas & TAS [1] \\
\hline MIGS-5 & Sample collection time & not reported & \\
\hline MIGS- & & & \\
\hline $\begin{array}{l}4.1 \\
\text { MIGS- } \\
4.2\end{array}$ & Latitude / Longitude & $25.066 /-77.339$ & NAS \\
\hline $\begin{array}{l}\text { MIGS- } \\
4.3\end{array}$ & Depth & not reported & \\
\hline $\begin{array}{l}\text { MIGS- } \\
4.4\end{array}$ & Altitude & not reported & \\
\hline
\end{tabular}

Evidence codes - IDA: Inferred from Direct Assay (first time in publication); TAS: Traceable Author Statement (i.e., a direct report exists in the literature); NAS: Non-traceable Author Statement (i.e., not directly observed for the living, isolated sample, but based on a generally accepted property for the species, or anecdotal evidence). These evidence codes are from the Gene Ontology project [14]. If the evidence code is IDA, then the property was observed for a living isolate by one of the authors or an expert mentioned in the acknowledgments.

\section{Growth conditions and DNA isolation}

$S$. nassauensis strain LLR-40K-21 ${ }^{\mathrm{T}}$, DSM 44728, was grown in DSMZ medium 553 (GPHF Medium) [15] at $28^{\circ} \mathrm{C}$. DNA was isolated from 1-1.5 g of cell paste using Qiagen Genomic 500 DNA Kit (Qiagen, Hilden, Germany) with lysis modification DALM according to Wu et al. [16].

\section{Genome sequencing and assembly}

The genome was sequenced using a combination of Sanger and 454 sequencing platforms. All general aspects of library construction and sequencing can be found at the JGI website. 454 Pyrosequencing reads were assembled using the Newbler assembler version 1.1.02.15 (Roche). Large Newbler contigs were broken into 7,157 overlap- 
ping fragments of $1,000 \mathrm{bp}$ and entered into assembly as pseudo-reads. The sequences were assigned quality scores based on Newbler consensus q-scores with modifications to account for overlap redundancy and to adjust inflated q-scores. A hybrid 454/Sanger assembly was made using the phrap assembler (High Performance Software, LLC). Possible mis-assemblies were corrected with Dupfinisher or transposon bombing of bridging clones [17]. Gaps between contigs were closed by editing in Consed, custom primer walk or PCR amplification. A total of 308 Sanger finishing reads were produced to close gaps and to raise the quality of the finished sequence. The error rate of the completed genome sequence is less than 1 in 100,000 . The final assembly consists of 81,931 Sanger and 851,638 pyrosequence reads. Together all sequence types provided $40.0 \times$ coverage of the genome.

Table 2. Genome sequencing project information

\begin{tabular}{lll}
\hline MIGS ID & Property & Term \\
\hline MIGS-31 & Finishing quality & Finished \\
& & Two genomic libraries: 8kb pMCL200 \\
MIGS-28 & Libraries used & and fosmid pcc1Fos Sanger libraries. \\
& & One 454 pyrosequence standard library \\
MIGS-29 & ABI3730, 454 GS FLX \\
MIGS-31.2 & Sequencing platforms & $11.0 \times$ Sanger; 29× pyrosequence \\
MIGS-30 & Assemblers coverage & Newbler version 1.1.02.15, phrap \\
MIGS-32 & Gene calling method & Prodigal 1.4, GenePRIMP \\
& INSDC ID & CP001778 \\
& Genbank Date of Release & not yet \\
& GOLD ID & Gc01107 \\
& NCBI project ID & 19713 \\
& Database: IMG-GEBA & 2501939631 \\
MIGS-13 & Source material identifier & DSM 44728 \\
& Project relevance & Tree of Life, GEBA \\
\hline
\end{tabular}

\section{Genome annotation}

Genes were identified using Prodigal [18] as part of the Oak Ridge National Laboratory genome annotation pipeline, followed by a round of manual curation using the JGI GenePRIMP pipeline [19]. The predicted CDSs were translated and used to search the National Center for Biotechnology Information (NCBI) nonredundant database, UniProt, TIGRFam, Pfam, PRIAM, KEGG, COG, and InterPro databases. Additional gene prediction analysis and manual functional annotation was performed within the Integrated Microbial Genomes Expert Review (IMG-ER) platform [20].

\section{Genome properties}

The genome is 6,841,557 bp long and comprises one circular chromosome with a $68.1 \%$ GC content (Table 3 and Figure 3). Of the 6,450 genes predicted, 6,487 were protein coding genes, and 53 RNAs; One hundred eight pseudogenes were also identified. The majority of the protein-coding genes $(66.8 \%)$ were assigned a putative function while those remaining were annotated as hypothetical proteins. The properties and the statistics of the genome are summarized in Table 3. The distribution of genes into COGs functional categories is presented in Table 4.

Table 3. Genome Statistics

\begin{tabular}{lrr}
\hline Attribute & Value & \% of Total \\
\hline Genome size (bp) & $6,841,557$ & $100.00 \%$ \\
DNA Coding region (bp) & $6,296,517$ & $92.03 \%$ \\
DNA G+C content (bp) & $4,661,422$ & $68.13 \%$ \\
Number of replicons & 1 & \\
Extrachromosomal elements & 0 & \\
Total genes & 6,540 & \\
\hline
\end{tabular}


Table 3. Genome Statistics (cont.)

\begin{tabular}{lrr}
\hline Attribute & Value & \% of Total \\
\hline RNA genes & 53 & $0.81 \%$ \\
rRNA operons & 2 & \\
Protein-coding genes & 6,487 & $99.20 \%$ \\
Pseudo genes & 108 & $1.65 \%$ \\
Genes with function prediction & 4,368 & $66.79 \%$ \\
Genes in paralog clusters & 1,454 & $22.23 \%$ \\
Genes assigned to COGs & 4,215 & $64.45 \%$ \\
Genes assigned Pfam domains & 4,474 & $68.41 \%$ \\
Genes with signal peptides & 1,698 & $25.96 \%$ \\
Genes with transmembrane helices & 1,858 & $28.41 \%$ \\
CRISPR repeats & 4 & \\
\hline
\end{tabular}

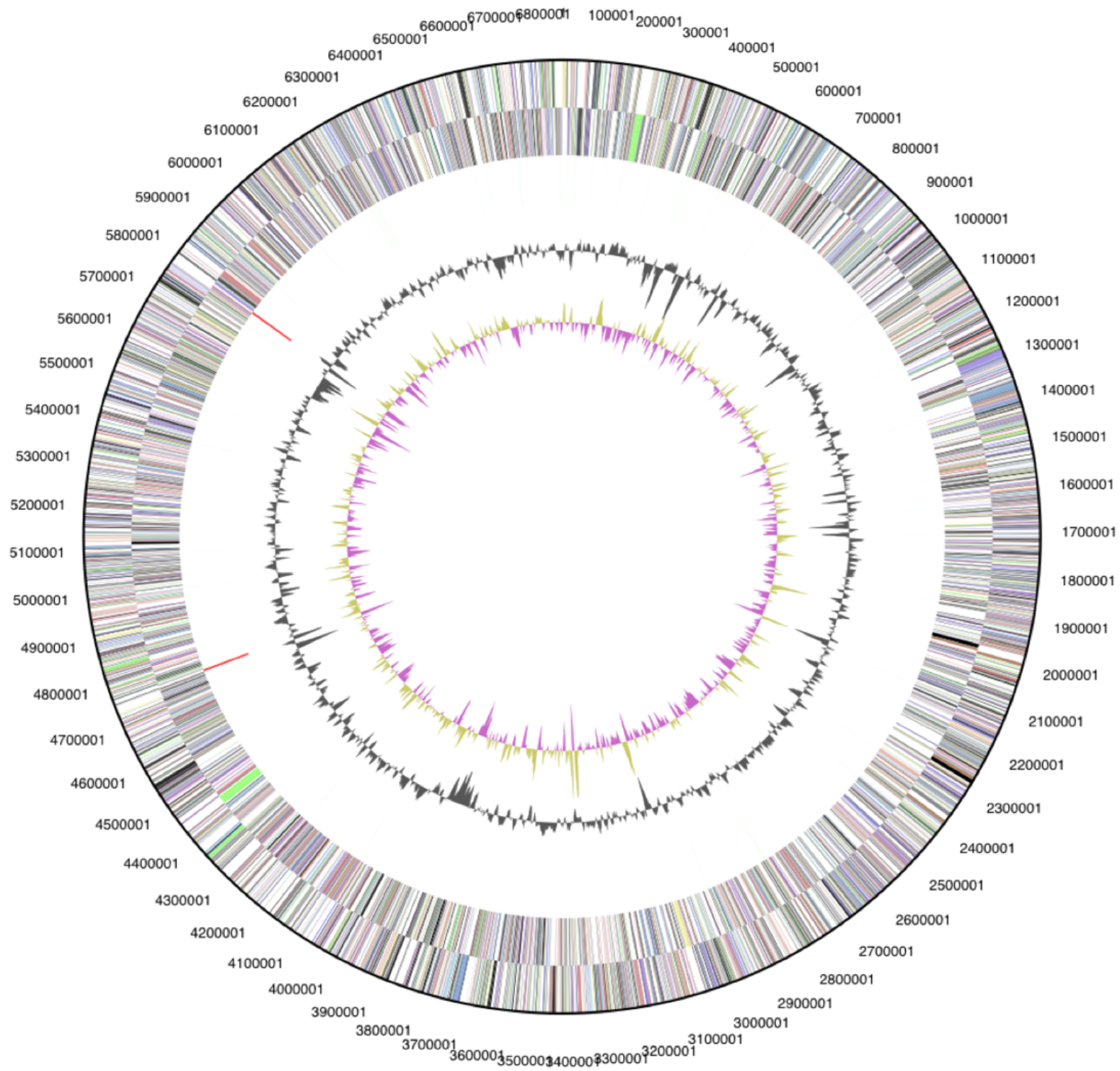

Figure 3. Graphical circular map of the genome. From outside to the center: Genes on forward strand (color by COG categories), Genes on reverse strand (color by COG categories), RNA genes (tRNAs green, rRNAs red, other RNAs black), GC content, GC skew. 
Table 4. Number of genes associated with the general COG functional categories

\begin{tabular}{lrrl}
\hline Code & value & \%age & Description \\
\hline J & 197 & 4.1 & Translation, ribosomal structure and biogenesis \\
K & 2 & 0.0 & RNA processing and modification \\
L & 653 & 13.5 & Transcription \\
D & 184 & 3.8 & Replication, recombination and repair \\
Y & 31 & 0.6 & Cell cycle control, mitosis and meiosis \\
V & 0 & 0.0 & Nuclear structure \\
T & 126 & 2.6 & Defense mechanisms \\
M & 348 & 7.2 & Signal transduction mechanisms \\
N & 214 & 4.4 & Cell wall/membrane biogenesis \\
Z & 2 & 0.0 & Cell motility \\
W & 1 & 0.0 & Cytoskeleton \\
U & 0 & 0.0 & Extracellular structures \\
O & 38 & 0.8 & Intracellular trafficking and secretion \\
C & 151 & 3.1 & Posttranslational modification, protein turnover, chaperones \\
G & 275 & 5.7 & Energy production and conversion \\
E & 436 & 9.0 & Carbohydrate transport and metabolism \\
F & 367 & 7.6 & Amino acid transport and metabolism \\
H & 102 & 2.1 & Nucleotide transport and metabolism \\
I & 229 & 4.7 & Coenzyme transport and metabolism \\
P & 178 & 3.7 & Lipid transport and metabolism \\
Q & 212 & 4.4 & Inorganic ion transport and metabolism \\
R & 169 & 3.5 & Secondary metabolites biosynthesis, transport and catabolism \\
S & 304 & 12.9 & General function prediction only \\
- & 2325 & 35.6 & Function unknown \\
\hline & & & \\
\hline
\end{tabular}

\section{Acknowledgements}

We would like to gratefully acknowledge the help of Susanne Schneider (DSMZ) for DNA extraction and quality analysis. This work was performed under the auspices of the US Department of Energy's Office of Science, Biological and Environmental Research Program, and by the University of California, Lawrence Berkeley National Laboratory under contract No. DE-

\section{References}

1. Labeda DP, Kroppenstedt RM. Stackebrandtia nassauensis gen. nov., sp. nov. and emended description of the family Glycomycetaceae. Int / Syst Evol Microbiol 2005; 55: 1687-1691. PubMeddoi:10.1099/ijs.0.63496-0

2. Wang $Y X$, Zhi XY, Zhang YQ, Cui XL, Xu LH, Li WJ. Stackebrandtia albiflava sp. nov. and emended description of the genus Stackebrandtia.
AC02-05CH11231, Lawrence Livermore National Laboratory under Contract No. DE-AC52-07NA27344, and Los Alamos National Laboratory under contract No. DEAC02-06NA25396. German Research Foundation (DFG) INST 599/1-1 supported DSMZ, and the Indian Council of Scientific and Industrial Research provided a Raman Research Fellow to Shanmugam Mayilraj.

Int J Syst Evol Microbiol 2009; 59: 574-577. PubMeddoi:10.1099/ijs.0.002147-0

3. Labeda DP, Testa A, Lechevalier MP, Lechevalier HA. Glycomyces, a new genus of the Actinomycetales. Int J Syst Bacteriol 1985; 35: 417-421.

4. Lee C, Grasso C, Sharlow MF. Multiple sequence alignment using partial order graphs. Bioinformat- 
iCs 2002; 18: 452-464.

PubMeddoi:10.1093/bioinformatics/18.3.452

5. Castresana J. Selection of conserved blocks from multiple alignments for their use in phylogenetic analysis. Mol Biol Evol 2000; 17: 540-552. PubMed

6. Stamatakis A, Hoover $\mathrm{P}$, Rougemont J. A rapid bootstrap algorithm for the RAxML web-servers. Syst Biol 2008; 57: 758-771. PubMeddoi:10.1080/10635150802429642

7. Liolios K, Mavromatis K, Tavernarakis N, Kyrpides NC. The Genomes OnLine Database (GOLD) in 2007: status of genomic and metagenomic projects and their associated metadata. Nucleic Acids Res 2008; 36: D475-D479. PubMeddoi:10.1093/nar/gkm884

8. Field D, Garrity G, Gray T, Morrison N, Selengut J, Sterk P, Tatusova T, Thompson N, Allen MJ, Angiuoli SV, et al. Towards a richer description of our complete collection of genomes and metagenomes: the "Minimum Information about a Genome Sequence" (MIGS) specification. Nat Biotechnol 2008; 26: 541-547.

PubMeddoi:10.1038/nbt1360

9. Woese CR, Kandler O, Wheelis ML. Towards a natural system of organisms: proposal for the domains Archaea, Bacteria, and Eucarya. Proc Natl Acad Sci USA 1990; 87: 4576-4579. PubMeddoi:10.1073/pnas.87.12.4576

10. Garrity GM, Holt JG. The Road Map to the Manual. In: Garrity GM, Boone DR, Castenholz RW (eds), Bergey's Manual of Systematic Bacteriology, Second Edition, Springer, New York, 2001, p. 119-169.

11. Stackebrandt E, Rainey FA, Ward-Rainey NL. Proposal for a new hierarchic classification system, Actinobacteria classis nov. Int J Syst Bacteriol 1997; 47: 479-491.

12. Zhi XY, Li WJ, Stackebrandt E. An update of the structure and $16 \mathrm{~S}$ rRNA gene sequence-based definition of higher ranks of the class Actinobacteria, with the proposal of two new suborders and four new families and emended descriptions of the existing higher taxa. Int I Syst Evol Microbiol 2009; 59: 589-608. PubMeddoi:10.1099/ijs.0.65780-0

13. Anonymous Biological Agents. Technical rules for biological agents www.baua.de TRBA 466.

14. Ashburner M, Ball CA, Blake JA, Botstein D, Butler $\mathrm{H}$, Cherry JM, Davis AP, Dolinski K, Dwight SS, Eppig JT, et al. Gene ontology: tool for the unification of biology. Nat Genet 2000; 25: 25-29. PubMeddoi:10.1038/75556

15. List of growth media used at DSMZ: http://www.dsmz.de/microorganisms/media_list.ph $\mathrm{p}$

16. Wu D, Hugenholtz P, Mavromatis K, Pukall R, Dalin E, Ivanova N, Kunin V, Goodwin L, Wu M, Tindall BJ, et al. A phylogeny-driven genomic encyclopedia of Bacteria and Archaea. Nature 2009; 462: 1056-1060. $\underline{\text { PubMeddoi:10.1038/nature08656 }}$

17. Sims D, Brettin T, Detter JC, Han C, Lapidus A, Copeland A, Glavina Del Rio T, Nolan M, Chen F, Lucas $\mathrm{S}$, et al. Complete genome sequence of $\mathrm{Ky}$ tococcus sedentarius type strain $\left(541^{\top}\right)$. Stand Genomic Sci 2009; 1: 12-20.doi:10.4056/sigs.761

18. Anonymous. Prodigal Prokaryotic Dynamic Programming Genefinding Algorithm. Oak Ridge National Laboratory and University of Tennessee 2009.

19. Pati A, Ivanova N, Mikhailova N, Ovchinikova G, Hooper SD, Lykidis A, Kyrpides NC. GenePRIMP: A Gene Prediction Improvement Pipeline for microbial genomes. (Submitted)

20. Markowitz VM, Mavromatis K, Ivanova NN, Chen IMA, Chu K, Kyrpides NC. Expert IMG ER: A system for microbial genome annotation expert review and curation. Bioinformatics 2009; 25: 2271-2278.

PubMeddoi:10.1093/bioinformatics/btp393 